\title{
Prevalence of respiratory disorders in veal calves and potential risk factors
}

\author{
M. Brscic, ${ }^{\star 1}$ H. Leruste,† L. F. M. Heutinck,‡ E. A. M. Bokkers,§ M. Wolthuis-Fillerup,‡ N. Stockhofe,‡ \\ F. Gottardo, ${ }^{*}$ B. J. Lensink, $†$ G. Cozzi, ${ }^{*}$ and C. G. Van Reenen $¥$ \\ *Department of Animal Medicine, Production and Health, University of Padova, Viale dell'Università 16, 35020 Legnaro (PD), Italy \\ †Groupe ISA, 48 boulevard Vauban, 59046 Lille Cedex, France \\ fLivestock Research, Wageningen UR, PO Box 65, 8200 AB Lelystad, the Netherlands \\ §Animal Production Systems, Wageningen UR, PO Box 338, $6700 \mathrm{AH}$ Wageningen, the Netherlands
}

\begin{abstract}
The study aimed to assess the in vivo and postmortem prevalence of respiratory disorders in veal calves and investigate risk factors associated with them. A cross-sectional study was carried out in 174 farms in the 3 major veal meat-producing countries in Europe (50 in France, 100 in the Netherlands, and 24 in Italy). Trained veterinarians visually evaluated individual calves of 1 batch per farm at 3 and 13 wk after arrival and at 2 wk before slaughter to assess the prevalence of hampered respiration, nasal discharge, and coughing. A random sample of lungs belonging to calves of the same batch was monitored at the slaughterhouse for mild to moderate or severe signs of pneumonia, and presence of pleuritis. Data regarding veal calf housing, feeding, and management and specific characteristics of the batch were collected through an interview with the stockperson, and the potential of these as respiratory disease risk factors was assessed. Regardless of the stage of fattening, the prevalence of in vivo signs of respiratory disorders in calves was always $<7 \%$. This low prevalence was likely the outcome of the general implementation by veal producers of standardized practices such as prophylaxis, all-in/all-out, and individual daily checks of the calves, which are recognized tools for effective disease prevention and management. However, at postmortem inspection, $13.9 \%$ and $7.7 \%$ of lungs showed mild to moderate and severe signs of pneumonia, respectively, and $21.4 \%$ of the inspected lungs had pleuritis. Thus, even mild clinical signs of respiratory disorder in calves at specific time points during the fattening period may be associated with high prevalence of lungs with lesions at slaughter. Alternatively, clinical symptoms recorded during routine visual inspections of veal calves on-farm may be poor predictors of the true prevalence of respiratory disease in calves. Among all potential risk factors considered,
\end{abstract}

Received July 7, 2011.

Accepted January 14, 2012.

${ }^{1}$ Corresponding author: marta.brscic@unipd.it those concerning the characteristics of the batch were predominant but factors related to housing, management and feeding equipment were also relevant. Different risk factors were involved at different stages of the fattening period. Therefore, to overcome respiratory disorders in veal calves, different solutions may apply to different stages of the fattening period.

Key words: veal calf, respiratory sign, risk factor, welfare

\section{INTRODUCTION}

Respiratory disorders are a relevant health problem in young cattle, especially those kept indoors (Virtala et al., 1999; Nikunen et al., 2007), and they affect both the health and welfare status of the animal per se and the economic results of the producers. Bovine respiratory disease is complex, characterized by many types of infection, each of them showing peculiar clinical signs and having different economic implications (Snowder et al., 2006). Main clinical signs include nasal discharge, coughing, fever, hampered respiration, inappetence, and depression. Signs may vary from subclinical to severe, which may lead to calf death. In addition to calf mortality and medical treatment costs, respiratory disorders imply economic losses due to slower growth of calves compared with healthy animals (Snowder et al., 2006). A compensatory gain over the entire 7-mo fattening period reduces the growth difference between healthy and infected calves in feedlots if the latter are effectively treated (Bateman et al., 1990). Moreover, costs are reported to be significantly greater when labor, calf isolation, increased time on feed, prophylaxis, and metaphylaxis treatments are considered (Snowder et al., 2006).

The etiology of respiratory disorders in young cattle is multifactorial, with different microbiological agents (bacteria, virus, mycoplasma) acting as initiating pathogens or as exacerbating and complicating factors in synergy with other factors mainly related to the rearing environment (Virtala et al., 1999; Arcangioli et al., 2008; Radaelli et al., 2008). Predisposing and causative 
factors are equally important because inhalation makes the respiratory system constantly exposed to the farm micro-climatic conditions. Most of the extrinsic risk factors impairing respiratory health are related to indoor housing: insufficient space allowance, overcrowded pens, unsuitable air temperature, quick temperature changes, high humidity, air dust, and high ammonia concentration (Lundborg et al., 2005). Therefore, farm micro-climate and environment should be appropriate to give calves a suitable aerial and thermal comfort (Cozzi et al., 2009).

Intrinsic predisposing factors are linked to the animal's innate and adaptive immunity. Veal calves are separated early from their dams, gathered from different farms, and transported to specialized fattening units when they are about 10 to $20 \mathrm{~d}$ old. Mixing calves from different farms during collection, transportation, and housing in a new environment exposes them to a heavy infection load including microorganisms against which they may not have colostral antibodies (Autio et al., 2007). Traditionally, veal calves were housed in individual crates for their entire life as a measure to minimize calf-to-calf contact for disease prevention. Today, the mandatory use of group housing imposed by the European regulation (European Council Directives 91/629/EC and 97/2 EC; European Council, 1991, 1997) has increased the risk of pathogen dissemination among calves. Veal farmers adopt several strategies to manage this problem. The all-in/all-out system is a biosecurity tool for the control of infectious diseases that implies the cleaning and disinfection of the empty barn before restocking. As an additional preventive measure, several veal units use individual separators (baby-box) during the first 6 to $8 \mathrm{wk}$ of fattening (European Council Directives 91/629/EC and 97/2 EC; European Council, 1991, 1997) to prevent quick spreading of pathogens and cross-sucking (Virtala et al., 1999).

Several studies have investigated the prevalence of respiratory disease in preweaned calves focusing mainly on dairy calves (Sivula et al., 1996; Virtala et al., 1996, 1999) and young beef cattle (Thomas et al., 2002; Hägglund et al., 2007). The main goal of these studies was to identify the pathogens through bacteriological and serological surveys. Incidence and etiology of respiratory diseases were also monitored in nonweaned calves by several authors (Assié et al., 2004; Autio et al., 2007; Arcangioli et al., 2008). No studies are available on the in vivo and postmortem prevalence of respiratory disorders in veal calves and the associated risk factors. The current study aimed to investigate the risk factors for the occurrence of problems at the respiratory system in veal calves, through a cross-sectional study carried out in a large sample of farms in the 3 major veal-producing countries in Europe.

\section{MATERIALS AND METHODS}

\section{Farm Sample}

To reflect a cross-section of European veal production, the prevalence of respiratory disorders in veal calves was assessed on 174 farms located in the 3 main veal-producing countries in Europe. One-hundred veal farms were monitored in the Netherlands, 50 in France, and 24 in Italy; the sample of farms was chosen to reflect the proportions of the prevailing rearing systems operating in Europe in terms of type of housing (small or large groups) and milk replacer delivery systems (bucket, trough, or automatic feeding device for individual teat feeding). Further details of the sample of farms and husbandry are described in Brscic et al. (2011).

\section{On-Farm Clinical Examination of the Calves}

The assessment protocol for examination of calf health status consisted of 3 visits carried out by trained veterinarians on 1 batch per farm. A batch was considered as a group of calves that arrived at the farm at the same time to be fattened and slaughtered together. Arrivals of the tested batches at the fattening units were distributed across all 4 seasons. Three visits were planned at different stages of the fattening period: the first at about 3 wk after arrival (early stage), the second at almost $13 \mathrm{wk}$ of the fattening period (middle stage), and the third at about 2 wk before slaughter (final stage). During each visit, the veterinarian visually assessed the respiratory status of 200 to 300 calves/ farm, avoiding any kind of animal manipulation. The veterinarian recorded the number of animals with evidence (presence/absence as binary measure) of respiratory disorders, including (1) hampered respiration (difficult or labored breathing, calf performing belly movements, increased respiratory frequency) indicative of acute lower airway disorders; (2) coughing (visible, sudden, and noisy expulsion of air) indicative of acute or chronic lower and/or upper airway disorders; and (3) nasal discharge (clearly visible flow/discharge from the nostrils, transparent to yellow/green and often of thick consistency) indicative of upper airway disorders.

\section{Farm Environment and Management Data}

Specific information regarding veal calf housing, feeding plan, and management, along with some distinctive characteristics of the tested batch, were collected through an interview with the stockperson (Table 1). The interviewer used a specific questionnaire developed to obtain this information in a short time avoiding their 
direct collection by the veterinarian. These data were considered variables potentially associated with an increased hazard of disease in the risk factor analysis (Table 1).

\section{Postmortem Inspection}

At the slaughterhouse, a random sample of about 100 pairs of lungs belonging to 100 calves of each batch observed during the fattening period were inspected to assess signs of lung disorders. Prior to the regular veterinary inspection, a trained examiner visually and manually examined both lungs and recorded those showing mild or moderate signs (presence of one large or several small spots of grey-red discoloration involving less than 1 lobe) or severe signs of pneumonia (presence of a grey-red discoloration area involving at least 1 full lobe or presence of abscesses, or both) on at least one side of the lungs. Involvement of the pleura by signs of pleuritis (presence of fibrinous attachment) was recorded as binary measure after assessing the whole lungs.

\section{Training}

To ensure that accurate and comparable observations were obtained, all examiners involved in clinical or pathological examinations in the 3 countries were subjected to a rigorous joint training prior to the start of the actual assessments on-farm or at the slaughterhouse. The training comprised theoretical and practical parts. During the theoretical part, trainees assessed sets of photos and videos with a known score (gold standard). Each trainee had to pass a final exam and reach at least $80 \%$ agreement with the gold standard (binomial test, $P<0.001$ ). The practical part considered the clinical assessment of live animals on-farm, and the in-line assessment of lungs at the slaughterhouse. Trainees teamed up with an experienced examiner, and a trainee was assumed to have successfully passed the practical test when the percentage of agreement with the experienced examiner was at least $80 \%$ out of 40 successfully assessed calves or lungs.

\section{Data Processing and Risk Factor Analysis}

Raw data from clinical examination of calves and postmortem inspection of lungs were expressed as percentages of observations and analyzed using GenStat (GenStat Committee, 2000), with farm as experimental unit. Prevalences of respiratory disorders were considered as response variables $(\mathrm{Y})$, and the explanatory variables $(\mathrm{X})$ were the potential risk factors obtained from the questionnaire as listed in Table 1. The dis- tribution of farms for each continuous risk factor (e.g., covariable, such as farm size or total amount of solid feed) included in the dataset was displayed with the use of histograms. Subsequently, all covariables were transformed into class variables (Table 1 ). In case of normally distributed covariables, thresholds for classes were chosen mainly according to location parameters such as lower, median, and upper quartile. For nonnormally distributed covariables, thresholds were arbitrarily chosen in such a way that sufficient numbers of farms were present in each class. In the case of the space allowance covariable, a cut-off point equal to the minimum legal requirement of $1.8 \mathrm{~m}^{2} /$ calf for calves with a BW $>220 \mathrm{~kg}$ (European Council Directive 97/2 EC; European Council, 1997) could be conveniently applied. Risk factor analyses were carried out separately for each respiratory disorder recorded in vivo at each of the 3 stages of fattening and for all postmortem measurements (\% lungs with mild/moderate signs, \% lungs with severe signs, and \% lungs with pleuritis), regardless of the possible correlations that could be found between them. Correlations between in vivo and postmortem signs were statistically significant but moderate to low (H. Leruste, unpublished data).

Potential risk factors were at first inspected individually adopting generalized linear model univariate analyses, and those significantly associated with the dependent animal measurement for $P<0.10$ were further included in the multivariate analysis. A two-way ANOVA was then carried out to test, for each pair of risk factors, the significance of main effects and their interaction and to evaluate potential multicollinearity between risk factors through the inspection of the distribution of farms across combinations of risk factors and calculation of the variance inflation factor. High variance inflation factors for the dummy variables for discrete risk factors may point to multicollinearity, and partial confounding between risk factors was automatically signaled. Selection of risk factors was performed both by stepwise forward and stepwise backward selection. The criterion in backward and forward selection was the adjusted $R^{2}$. On the union of final models of both selection procedures, best subset selection was performed and significance tests for the effects of the selected risk factors were evaluated. Only risk factors that added significance $(P<0.05)$ to the model were retained. Consistent with the methodological approach used by Somers et al. (2005) and Cramer et al. (2009) in previous risk analysis on dairy cattle, risk factors that were (partially) confounded were not simultaneously maintained in the same model and only main effects were considered without interactions.

Because the response variables were always prevalences; that is, percentages per farm (batch), logistic 
Table 1. Potential risk factors recorded and tested for their association with respiratory disorders in veal calves

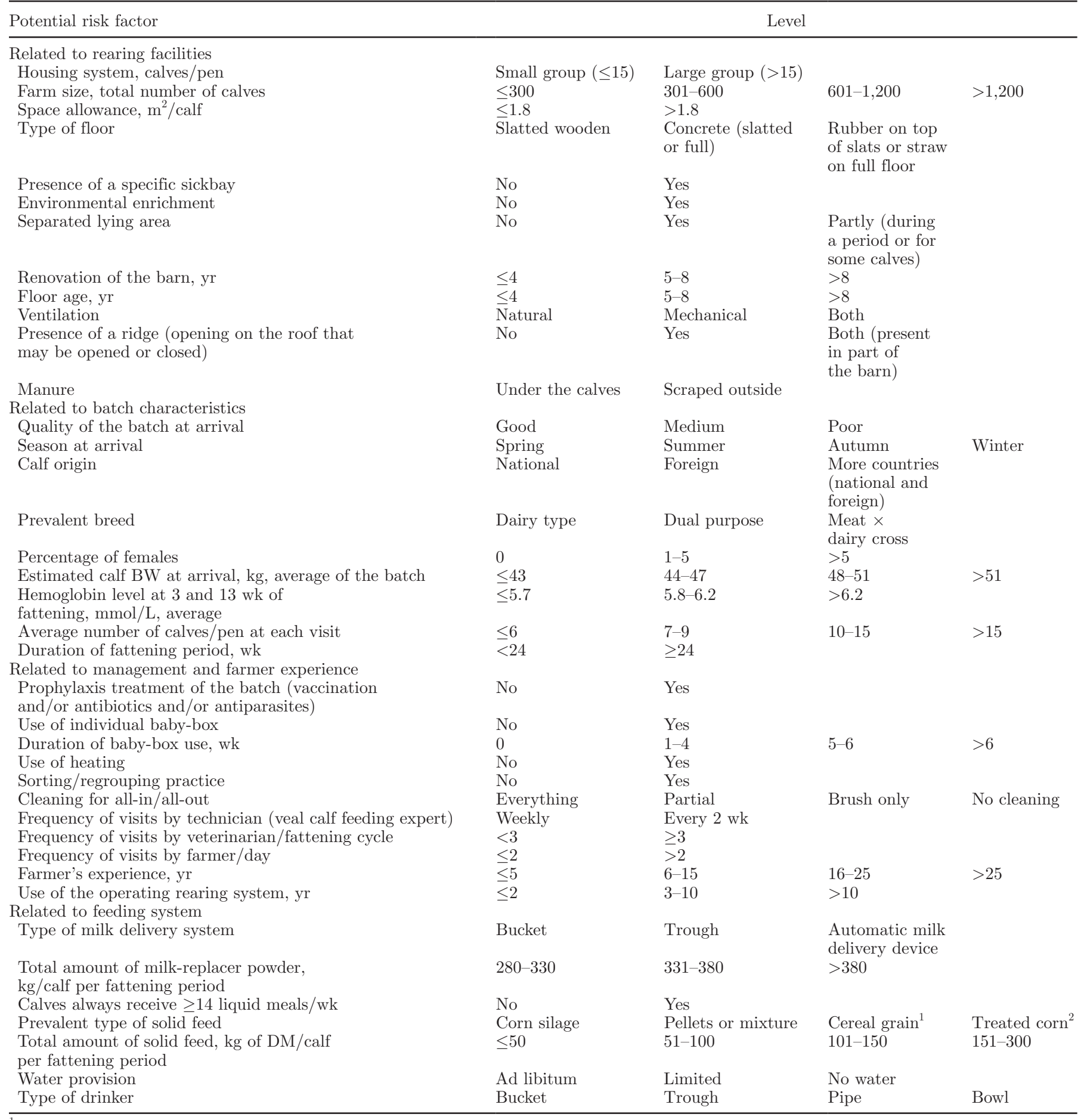

${ }^{1}$ Barley or corn.

${ }^{2}$ Rolled or flaked corn, or both.

regression models were used, specifying a binomial variance function with a multiplicative dispersion parameter. Estimation was by quasi likelihood. The dispersion parameter in the variance was estimated from the data
(McCullagh and Nelder, 1984). Soundness of the model was assessed according to the normal distribution of the residuals. Predicted prevalence, odds ratio, and the 95\% CI were obtained, and the lowest predicted preva- 
lence of a given problem was used as the reference value for the pairwise comparison between levels.

\section{RESULTS AND DISCUSSION}

\section{Observed Batch Size and Farm Characteristics}

The average size of the evaluated batch was $252 \pm 77$ (SD) calves, and about $32 \%$ of calves were followed at the slaughterhouse, with an average of $138 \pm 59$ (SD) pairs of lungs inspected per batch.

The majority of farms included in the sample $(149 / 174,86 \%)$ had a group size of fewer than 16 calves/pen, most (73\%) had fewer than 7 calves/pen, whereas the remaining farms had a group size of more than 15 calves/pen. The study was dominated by male dairy-bred calves, reared throughout the year: $70 \%$ of batches had less than $5 \%$ heifers, and farms raising dualpurpose and crossbred calves comprised $16.5 \%$ and $17 \%$ of the total, respectively. Farm size varied across the sample and 4 categories were identified: $22 \%$ of farms reared $\leq 300$ calves, $29 \%$ reared 301 to 600 calves, $35 \%$ reared 601 to 1,200 calves, and $13 \%$ of farms reared $>1,200$ calves per year. The fattening period lasted an average of $25.8 \pm 3.7$ (SD) wk.

Seventy-nine percent of farms complied with the legal requirement for space allowance of $1.8 \mathrm{~m}^{2} /$ calf for a calf weighing $>220 \mathrm{~kg}$, and $21 \%$ of farms exceeded the space allowance for the entire fattening period. Regarding the type of pen floor, $79 \%$ of the farms had fully slatted wooden floors, $15 \%$ had concrete floors (slatted or solid), and only $6 \%$ of the farms used rubber-covered slatted or straw-bedded floors. None of the farms offered calves access to an outdoor area. Individual baby-boxes to separate pen-mates in the early stage of fattening were used on $83 \%$ of the farms. Calves were regrouped during fattening on $94 \%$ of farms to harmonize growth within pen.

\section{Prevalence of Respiratory Disorders Recorded In Vivo and at Postmortem Inspection}

Prevalence of respiratory disorders recorded during the 3 clinical visits are shown in Figure 1; among the 3 criteria considered, nasal discharge always showed an incidence of $5 \%$ or higher. Hampered respiration and coughing were less frequently observed and their prevalence tended to decrease as calves aged. The prevalence of these 2 disorders at $3 \mathrm{wk}$ of fattening could arise from the common practice of commingling calves originating from different dairy herds in a single batch. These very young animals may not have antibodies to counteract the spread of diseases. The prevalence of hampered respiration showed a large reduction in the later stages of the fattening, likely because of the metaphylaxis provided by the farmers. Farmers consider this clinical sign to be related to a severe respiratory disorder and are likely to promptly treat calves exhibiting this sign (Callan and Garry, 2002; Assié et al., 2004). Recorded prevalences of hampered respiration at $13 \mathrm{wk}$ of fattening and at $2 \mathrm{wk}$ before slaughter were too low to allow us to conduct a risk factor analysis. On the contrary, nasal discharge and coughing are not considered signs of severe disease; therefore, farmers likely do not seek specific pharmacological treatments until a large part of the batch is involved or when single calves show signs of severely impaired health. Respiratory disorders are reported within the main causes of mortality in dairy calves (Virtala et al., 1996; Svensson et al., 2006), and the progressive decline of hampered respiration and coughing could be related to calf mortality. The cumulative average values for mortality recorded in this study were 1.0, 2.1, and $3.1 \%$ of calves by wk 3 and 13 of fattening and 2 wk before slaughter, respectively.

In this study, the signs of respiratory disorders recorded in vivo showed a generally low incidence compared with the outcomes of studies carried out on replacement dairy calves (Virtala et al., 1996; Nikunen et al., 2007). This might be due to a combination of 2 factors. The first is the use of pharmaceutical treatments at the group level implemented in veal production to control disease outbreak that could cause apparent recovery with decline of symptoms. The second is the different assessment methodology used for group-housed veal calves compared with that of replacement dairy calves, whose housing conditions differ (Virtala et al., 1996; Lago et al., 2006; Nikunen et al., 2007).

Prevalence of signs of respiratory diseases recorded postmortem was moderately high: on average 13.9 and $7.7 \%$ of lungs had mild to moderate and severe

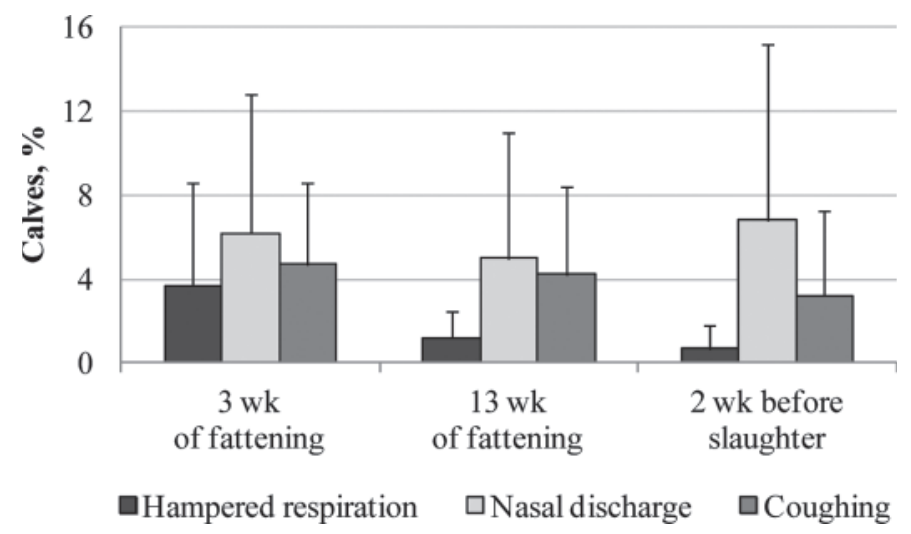

Figure 1. Prevalence of respiratory disorders as a percentage of observed calves (mean $\pm \mathrm{SD}$ ) in 174 veal calf farms in 3 stages of the fattening period. 
signs of pneumonia, respectively, and $21.4 \%$ of lungs exhibited signs of pleuritis. The apparent difference between prevalences obtained at the in vivo examination and the postmortem inspection in the present study suggests that even mild clinical signs of respiratory disorders in veal calves at specific time points during the fattening period may be associated with a high prevalence of lungs with lesions at slaughter, perhaps because silent pathogens persisted in clinically healthy animals, as observed by Jaeger et al. (2007). Therefore, clinical symptoms scored during routine visual inspections of veal calves on-farm may be poor predictors of the true prevalence of respiratory disease in calves. In our study, on-farm clinical examinations took place only at specific time points; therefore, we were unable to determine the actual severity of episodes of respiratory disease (e.g., for how many days animals exhibited clinical signs). Moreover, episodes of respiratory disease outside the specific time points when clinical examinations took place were missed entirely. In contrast, lung lesions likely reflected the cumulative effect of all respiratory diseases experienced across the entire fattening period. It seems unlikely that clinical symptoms revert by time of slaughter or during transportation to the slaughterhouse because detected signs on lungs were chronic and transport of finished veal calves was shown not to compromise their health status (Grigor et al., 2004).

\section{Risk Factors for Respiratory Disorders}

Considering all potential risk factors for the occurrence of respiratory disorders in veal calves, the available literature is mainly addressed toward poor indoor housing conditions (Lundborg et al., 2005; EFSA, 2006; Cozzi et al., 2009). Some of the risk factors identified in the present study were linked to characteristics of the rearing facilities and to the equipment necessary to ensure a good farm environment but they were not predominant. Respiratory disorders were mainly associated with specific traits of the batch but also to farm management, the farmer's experience, and the feeding system. Practices that could be related to the control of respiratory disease outbreaks such as prophylaxis, cleaning for all-in/all-out, presence of a specific sickbay, and frequency of calf health checks did not show significant relevance. This might be due to the lack of variability between farms because these practices are commonly used in the majority of the veal-producing units and therefore they could have already efficiently reduced the prevalence of respiratory disorders, justifying their general low prevalence observed in vivo.

Specific batch characteristics, particularly the estimated $\mathrm{BW}$ of the calves at arrival at the fattening unit, were risk factors for hampered respiration at the early stage of fattening (Table 2). Calves arriving at the farm with lower BW showed a higher risk of hampered respiration compared with heavier calves at the 3 -wk assessment. This was likely due to a poorer energy or immunological status impairing their ability to cope with the receiving environment of new housing facilities, pen-mates, stockmen, and management (Miller et al., 1980; Autio et al., 2007). However, a lower BW was not a significant risk factor for hampered respiration at subsequent inspections. It is possible that farmers promptly treated the lightest and weakest calves, determining their quick recovery and this would justify the reduction of hampered respiration prevalence at the middle and end of the fattening period.

Batches judged by the farmer to be good quality at arrival showed an increased risk of nasal discharge at the end of the fattening (Table 3). A skilled stockperson should guarantee constant attention toward all animals throughout the fattening period, regardless of their general appearance and BW at arrival. Gulliksen et al. (2009) reported that risk of respiratory disorders might increase with reduced individual inspection of calves. In our study, a higher prevalence of hampered respiration in young calves was observed when the number of calves/pen increased (Table 2), likely due to less accurate control of individual calves' health. The quick detection of sick calves by the stockperson during milk feeding is probably delayed in larger groups, allowing the spread of pathogen among pen-mates (Lundborg et al., 2005; Svensson and Liberg, 2006).

In dairy cattle, increased risk for respiratory disorders is reported for larger compared with smaller dairy herds (Norström et al., 2000). In the current study, in the early stage of fattening, the risk of nasal discharge increased as farm size decreased. On the contrary, at the end of the fattening period, the risk was lowest in small- to mid-size farms (301 to 600 calves), and this farm size seemed a better option for controlling the prevalence of respiratory disorders (Table 3). However, according to results reported in the literature, factors such as prophylaxis, disease management, and particularly the animal:stockperson ratio, rather than farm size, play a relevant role in the prevalence of respiratory disorders in calves (Waltner-Toews et al., 1986; Assié et al., 2004; Lundborg et al., 2005). In this study, the frequency of calf health checks carried out by the farmer was not relevant in the control of the disease, but frequency of veterinarian visits did influence the prevalence of respiratory disorders. Fewer visits by the veterinarian throughout the fattening period increased the risk of coughing at the end of the period (Table 4). The farmer's skills were shown to be important, because calves reared by farmers who had been using 
Table 2. Multivariate regression model for occurrence of hampered respiration in veal calves at 3 wk of fattening (adjusted $\mathrm{R}^{2}=25 \%$ )

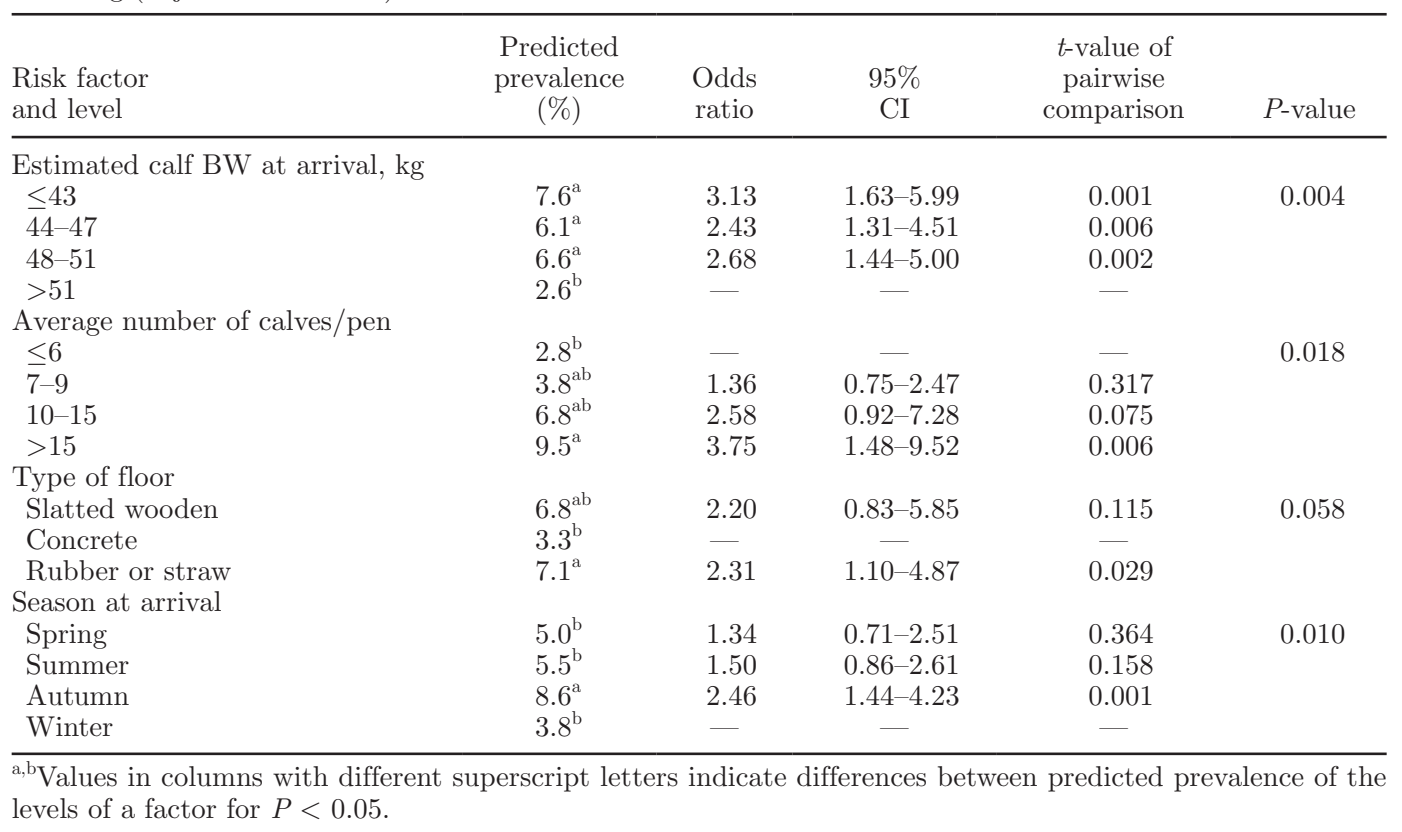

the housing and feeding system for a shorter period had a higher risk of occurrence of nasal discharge at $13 \mathrm{wk}$ (Table 3) and of mild pneumonia postmortem (Table 5) compared with those reared by farmers with more than 10 yr of experience. Timely supervision by the farmer and the veterinarian is likely to allow quicker detection of respiratory problems and earlier treatment of sick calves. Gathering information from farm health records could have allowed us to validate this hypothesis, but consultation of farm records is not always easy and this was the reason not to implement it in the current study.

The use of individual baby-boxes and the duration of their use (legal up to 8 wk of age) have shown to be relevant management factors for the prevention of calf respiratory disorders. As expected, the use of individual baby-boxes in the early stage of the fattening period reduced the prevalence of nasal discharge (Table 3) and coughing (Table 4), likely by reducing contact among neighboring calves. This is in agreement with results reported for dairy calves within $90 \mathrm{~d}$ of age by Virtala et al. (1999) and Svensson et al. (2003). It is interesting to notice that prolonged use of these individual separators over $4 \mathrm{wk}$ was a risk for nasal discharge at $13 \mathrm{wk}$ (Table 3) and their use for 5 to 6 wk was a risk factor for coughing at the end of the fattening period (Table 4). Perhaps prolonged forced isolation from pen-mates acted as a chronic stressor due to the lack of opportunity to perform social behaviors, resulting in immunosuppression (Glaser and Kiecolt-Glaser, 2005). However, this hypothesis needs to be confirmed in other studies.
The season of arrival at the fattening units influenced most respiratory disorders (Tables 2 to 5), confirming that the outcome of these diseases is closely related to the farm micro-climatic conditions (Virtala et al., 1999; Arcangioli et al., 2008; Radaelli et al., 2008). A higher risk of hampered respiration was observed for calves that arrived at the fattening unit during autumn compared with those housed in winter (Table 2), despite a greater expectation of respiratory disorders in the latter season (Diesel et al., 1991; Lundborg et al., 2005; Lago et al., 2006). Calves housed in autumn could have received weakened passive immunity from their dams due to the dams' exposure to summer heat stress during late pregnancy (Callan and Garry, 2002). Although it was clear that the season at arrival affected nasal discharge at 13 wk of fattening (Table 3), coughing $2 \mathrm{wk}$ before slaughter (Table 4), and the prevalence of severe pneumonia recorded postmortem (Table 5), the time between the outset of the fattening and the observation session should be considered. Indeed, calves housed in spring, spending the warm season in the barn, had lower risk of developing respiratory problems compared with calves housed later in the year. We observed a clear association of an increased risk of nasal discharge and postmortem signs of pneumonia with cold season arrival, which confirms the results obtained by Lago et al. (2006) and underlines that environmental temperature is highly relevant when discussing respiratory problems prevalence, even in standardized indoor housing systems such as those used for veal calves. Although 
Table 3. Multivariate regression model for occurrence of nasal discharge in veal calves at 3 wk of fattening (adjusted $\mathrm{R}^{2}=31 \%$ ), 13 wk of fattening (adjusted $\mathrm{R}^{2}=39 \%$ ), and 2 wk before slaughter (adjusted $\mathrm{R}^{2}=46 \%$ )

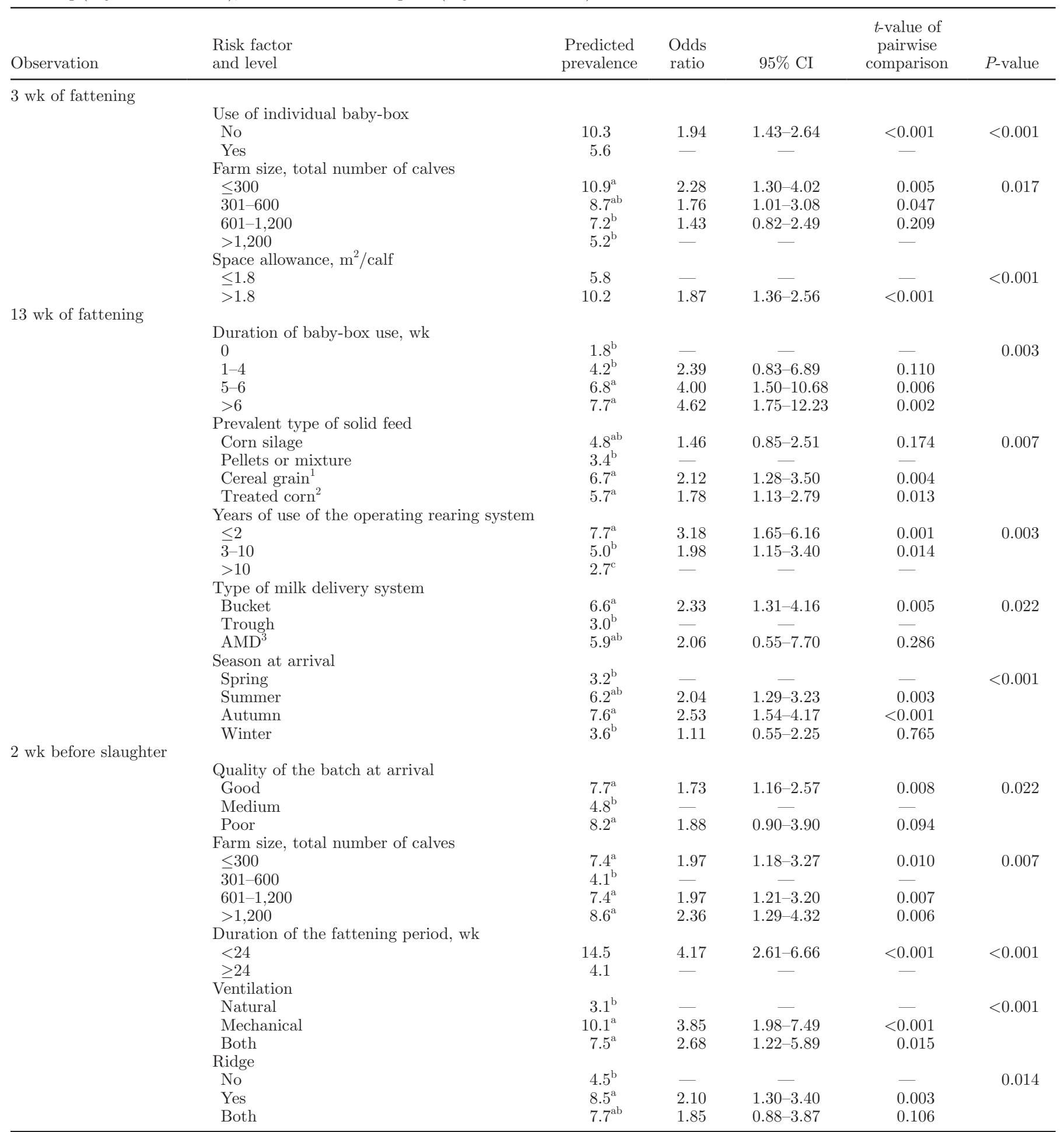

\footnotetext{
${ }^{\mathrm{a}-\mathrm{c}}$ Values in columns with different superscript letters indicate differences between predicted prevalence of the levels of a factor for $P<0.05$. ${ }^{1}$ Barley or corn.

${ }^{2}$ Rolled or flaked corn, or both.

${ }^{3}$ Automatic milk delivery device.
} 
Table 4. Multivariate regression model for occurrence of coughing in veal calves at 3 wk of fattening (adjusted $\mathrm{R}^{2}=16 \%$ ), 13 wk of fattening (adjusted $\mathrm{R}^{2}=36 \%$ ), and 2 wk before slaughter (adjusted $\mathrm{R}^{2}=43 \%$ )

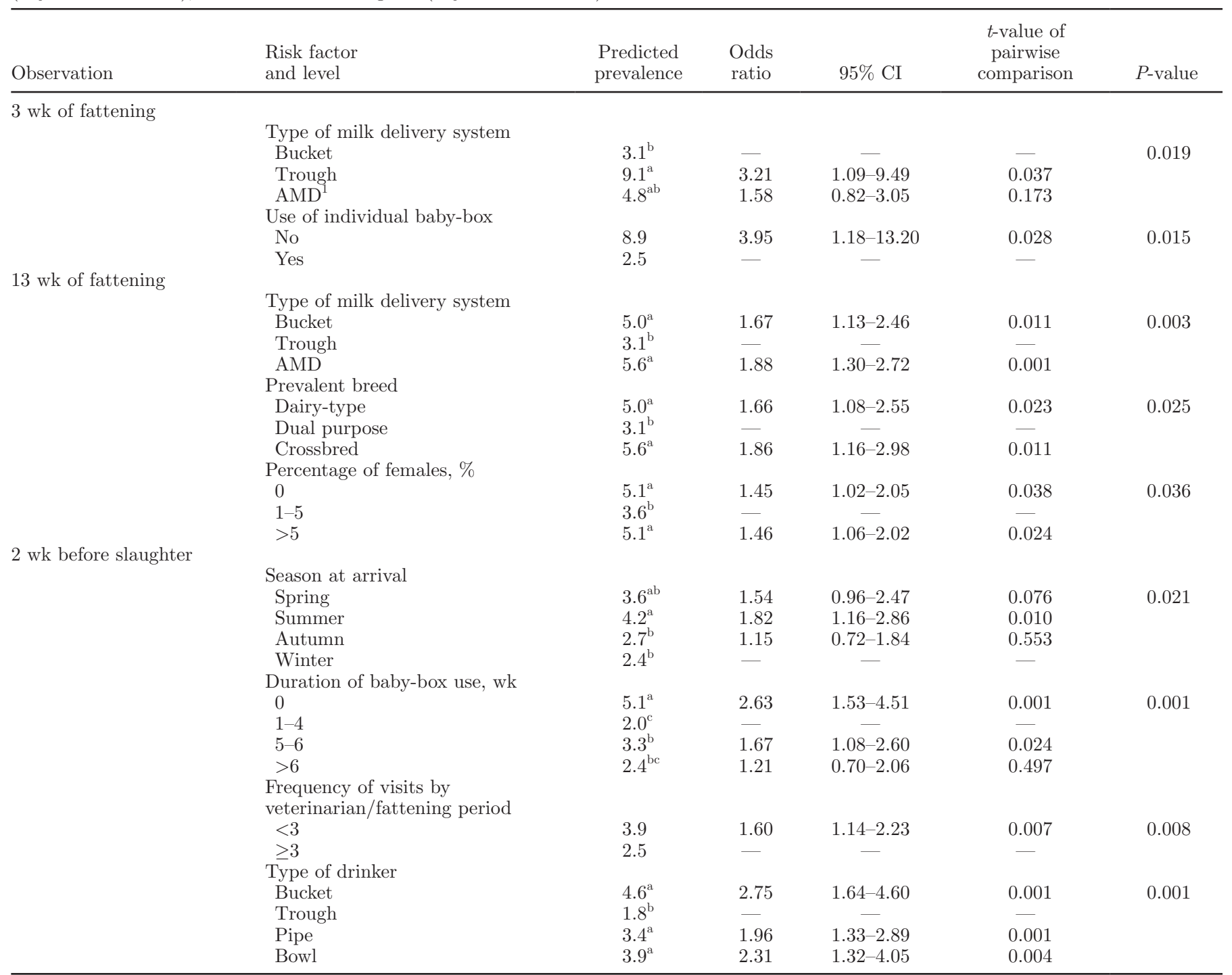

${ }^{\mathrm{a}-\mathrm{C}}$ Values in columns with different superscript letters indicate differences between predicted prevalence of the levels of a factor for $P<0.05$.

${ }^{1}$ Automatic milk delivery device.

the duration of the fattening cycle was also standardized, averaging about $25.8 \pm 3.7(\mathrm{SD})$ wk, contradicting results were observed, with the risk of nasal discharge increasing for calves finished in a shorter period (Table 3 ), whereas signs of pleuritis were associated with an extended finishing period (Table 5). The latter effect could be associated with slower growth in calves suffering from respiratory disease but no evident conclusions in this regard may be drawn. No firm conclusions can be made for the effects of breed and percentage of female calves, which were significant risks for the occurrence of coughing at 13 wk of fattening (Table 4). Snowder et al. (2006) reported that relatively small differences in resistance to bovine respiratory disease exist between breed types and that no strong evidence exists that composite breeds are less susceptible than purebreds to disease.

In the literature, factors related to the characteristics of the rearing facilities have been associated with respiratory disorders, indicating that the farm environment should be appropriate to provide calves a suitable aerial and thermal comfort (Cozzi et al., 2009). However, in the present study, some strategies usually applied to increase calf comfort did not yield the expected results, particularly in the early stage of fattening. A higher risk of hampered respiration was found in calves housed on rubber or straw compared with those housed on concrete floors (Table 2), and 
Table 5. Multivariate regression model for occurrence of mild or moderate signs of pneumonia (adjusted $\mathrm{R}^{2}=18 \%$ ), severe signs of pneumonia (adjusted $\mathrm{R}^{2}=18 \%$ ), and pleuritis in veal calves (adjusted $\mathrm{R}^{2}=42 \%$ )

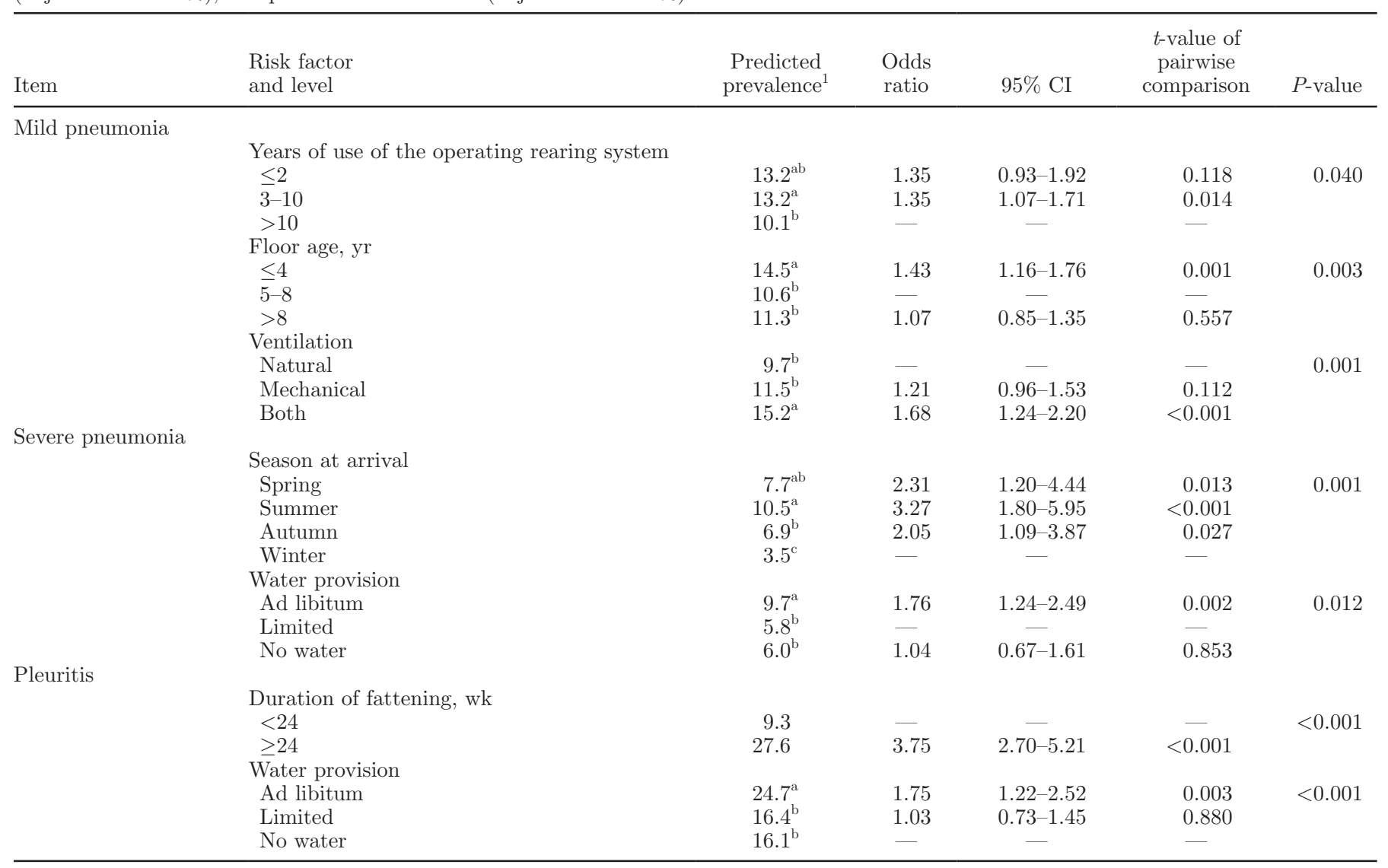

\footnotetext{
${ }^{a-c}$ Different superscript letters in columns indicate difference between predicted prevalence of the levels of a factor for $P<0.05$.
}

housing animals with a space allowance $>1.8 \mathrm{~m}^{2} /$ calf increased the risk of occurrence of nasal discharge compared with a lower space allowance (Table 3). The increased prevalence of respiratory signs when calves were housed on straw-bedded floors was likely due to dust inhalation (Takai et al., 1998). However, straw and rubber flooring solutions are not frequently applied in veal fattening units even though they offer better thermal comfort and avoid potential health risks for calves exposed to a cold environment (Hänninen et al., 2003). A lesser space allowance at the onset of the fattening acting as a preventive measure suggests that calves of very young age cope well in a constrained environment. A limited space allowance may reduce animal activity, thus decreasing exposure to dust and airborne pathogens that have adverse effects on both mucociliary clearance and respiratory defense mechanisms (Callan and Garry, 2002). We speculate that a higher concentration of noxious chemical constituents in new wooden floors treated with protective urethane or varnish might explain newer floors (less than $4 \mathrm{yr}$ ) being risk factors for mild pneumonia signs (Table
5). However, other factors may have been confounded with age of the floor that caused this effect.

Prevalence of respiratory disorders was decreased for calves housed in barns with natural compared with mechanical ventilation or a combination of the two, making the presence of mechanical ventilation a risk factor for nasal discharge at the end of the fattening (Table 3) and mild pneumonia signs (Table 5). The presence of a ridge on the roof of the barn to allow air circulation was a risk factor for nasal discharge in the late stage of the fattening (Table 3). This underlines that ventilation is very important for the prevention of respiratory disease in cattle, but it also suggests that calves are very susceptible to drafts. Lundborg et al. (2005) reported an increased risk for respiratory sounds at lung auscultation in calves exposed to draft, whereas they were less likely on farms where ventilation was natural and where the lack of the ridge did not allow air exit from the roof.

Factors related to the feeding system were significantly associated with occurrence of respiratory disorders. In the early stage of fattening, a common trough 
increased the risk of coughing (Table 4), whereas at the later assessments, it reduced the prevalence of both nasal discharge and coughing (Tables 3 and 4). Perhaps, at an early stage of fattening, when calves are not fully adapted to the new rearing environment, the higher drinking speed allowed by trough feeding along with the absence of teats might increase the risk of accidental inhalation of milk-replacer into the airways, stimulating coughing. In the later stage of fattening, the positive effect of trough feeding on reducing the prevalence of respiratory disorders signs could be related to improved hygienic conditions. The proper cleaning of individual buckets is more time- and labor-consuming than that of either troughs or automatic milk delivery devices. A similar hypothesis might be drawn also for drinking water delivery systems. Compared to the trough, buckets, pipes, and bowls acted as risk factors for coughing, likely because of their lower level of cleanliness and hygiene (Table 4).

Unexpectedly, ad libitum availability of drinking water significantly increased severe signs of pneumonia and pleuritis (Table 5). Drinking water is an important issue for calf welfare because, rather than covering a shortage in calf water requirement, it prevents the arousal of nonnutritive oral behaviors (Gottardo et al., 2002). In our study, this risk factor might be related to drinking behavior because it is likely that, when water is constantly available, calves play with the drinker, wasting the water and wetting the pen floor, and penmates lay underneath the drinkers. Calves exposed to a moist and cold environment are more sensitive to respiratory problems (Diesel et al., 1991).

The amounts of milk replacer powder and solid feed provided throughout the fattening period, which were highly relevant risk factors for gastrointestinal problems (Brscic et al., 2011), did not affect respiratory disorders. Type of solid feed, however, was associated with nasal discharge; in particular, calves fed cereal grain or treated corn showed a higher prevalence compared with those receiving pellets or mixtures. This might be due to the provision of high-starch solid feeds negatively interfering with calf health. Donovan et al. (2003) showed that the immune response of calves fed an acidogenic diet is weakened, increasing their susceptibility to other pathogens.

\section{CONCLUSIONS}

The present paper investigated the prevalence of respiratory disorders and associated risk factors in veal calves in a large sample of farms in the main veal-producing countries of Europe. The prevalences detected in vivo were low but the results from postmor- tem lung inspection suggested that even mild clinical signs of respiratory disorders during fattening should not be neglected. Different risk factors related to batch characteristics, housing, management, and feeding were relevant at different observation time points and, in some cases, controversial findings were observed. This excludes the possibility of carrying out a single visit to a farm or slaughterhouse to identify both problems and risks. Moreover, it is unrealistic to suggest a unique solution to overcome all respiratory disorders. Continuous monitoring of calf health by an experienced farmer, along with repeated herd screenings at strategic timepoints by a veterinarian, seems to be the best strategy to plan time-effective interventions to manage disease outbreaks.

\section{ACKNOWLEDGMENTS}

The study was carried within the activity of the European Union-funded integrated project FOODCT-2004-506508 "Welfare Quality: Science and society improving animal welfare in the food quality chain" and was co-financed by the Dutch, French, and Italian veal producers. The text represents the authors' views and does not necessarily represent a position of the Commission, who will not be liable for the use made of such information. The authors thank all the veal integrators and farmers who took part in the study.

\section{REFERENCES}

Arcangioli, M. A., A. Duet, A. Meyer, G. Dernburg, A. Bézille, P. Poumarat, and F. Le Grand Dominique. 2008. The role of Mycoplasma bovis in bovine respiratory disease outbreaks in veal calf feedlots. Vet. J. 177:89-93.

Assié, S., H. Seegers, and F. Beaudeau. 2004. Incidence of respiratory disorders during housing in non-weaned Charolais calves in cow-calf farms of Pays de la Loire (western France). Prev. Vet. Med. 63:271-282.

Autio, T., T. Pohjanvirta, R. Holopainen, U. Rikula, J. Pentikäinen, A. Huovilainen, H. Rusanen, T. Soveri, L. Sihvonen, and S. Pelkonen. 2007. Etiology of respiratory disease in non-vaccinated, non-medicated calves in rearing herds. Vet. Microbiol. 119:256-265.

Bateman, K. G., W. Martin, P. E. Shewen, and P. I. Menzies. 1990. An evaluation of antimicrobial therapy for undifferentiated bovine respiratory disease. Can. Vet. J. 31:689-696.

Brscic, M., L. F. M. Heutinck, M. Wolthuis-Fillerup, N. Stockhofe, B. Engel, E. K. Visser, F. Gottardo, E. A. M. Bokkers, B. J. Lensink, G. Cozzi, and C. G. Van Reenen. 2011. Prevalence of gastrointestinal disorders recorded at postmortem inspection in white veal calves and associated risk factors. J. Dairy Sci. 94:853-863.

Callan, R. J., and F. B. Garry. 2002. Biosecurity and bovine respiratory disease. Vet. Clin. North Am. Food Anim. Pract. 18:57-77.

Cozzi, G., M. Brscic, and F. Gottardo. 2009. Main critical factors affecting the welfare of beef cattle and veal calves raised under intensive rearing systems in Italy: A review. Ital. J. Anim. Sci. 8(Suppl. 1):67-80.

Cramer, G., K. D. Lissemore, C. L. Guard, K. E. Leslie, and D. F. Kelton. 2009. Herd-level risk factors for seven different foot lesions 
in Ontario Holstein cattle housed in tie stalls or free stalls. J. Dairy Sci. 92:1404-1411.

Diesel, D. A., J. L. Lebel, and A. Tucker. 1991. Pulmonary particle deposition and airway mucociliary clearance in cold-exposed calves. Am. J. Vet. Res. 52:1665-1671.

Donovan, D. C., A. R. Hippen, D. J. Hurley, and C. C. L. Chase. 2003. The role of acidogenic diets and $\beta$-hydroxybutyrate on lymphocyte proliferation and serum antibody response against bovine respiratory viruses in Holstein steers. J. Anim. Sci. 81:3088-3094.

EFSA (European Food and Safety Authority). 2006. The risk of poor welfare in intensive calf farming systems. An update of the Scientific Veterinary Committee Report on the Welfare of Calves. EFSA J. 366:1-36.

European Council. 1991. European Council Directive 91/629/EC, 11 December 1991. Off. J. L 340/28.

European Council. 1997. European Council Directive 97/2/EC, 28 January 1997. Off. J. L 25/24.

GenStat Committee. 2000. Reference Manual. R. G. Payne and G. M. Arnold, ed. Procedure Library PL12, VSN International, Oxford, UK.

Glaser, R., and J. K. Kiecolt-Glaser. 2005. Stress-induced immune dysfunction: Implications for health. Nat. Rev. Immunol. 5:243-251.

Gottardo, F., S. Mattiello, G. Cozzi, E. Canali, E. Scanziani, L. Ravarotto, V. Ferrante, M. Verga, and I. Andrighetto. 2002. The provision of drinking water to veal calves for welfare purpose. J. Anim. Sci. 80:2362-2372

Grigor, P. N., M. S. Cockram, W. B. Steele, J. McIntyre, C. L. Williams, I. E. Leushuis, and C. G. Van Reenen. 2004. A comparison of the welfare and meat quality of veal calves slaughtered on the farm with those subjected to transportation and lairage. Livest. Prod. Sci. 91:219-228.

Gulliksen, S. M., E. Jor, K. I. Lie, T. Løken, J. Åkerstedt, and O. Østerås. 2009. Respiratory infections in Norwegian dairy calves. J. Dairy Sci. 92:5139-5146.

Hägglund, S., M. Hjort, D. A. Graham, P. Öhagen, M. Törnquist, and S. Alenius. 2007. A six-year study on respiratory viral infections in a bull testing facility. Vet. J. 173:585-593.

Hänninen, L., H. Hepola, J. Rushen, A. M. De Passille, P. Pursiainen, V. M. Tuure, L. Syrjäläqvist, M. Pyykkönen, and H. Saloniemi. 2003. Resting behaviour, growth and diarrhoea incidence rate of young dairy calves housed individually or in groups in warm or cold building. Acta Agric. Scand. A Anim. Sci. 53:21-28.

Jaeger, J., E. Liebler-Tenorio, N. Kirschvink, K. Sachse, and P. Reinhold. 2007. A clinically silent respiratory infection with Chlamydophila spp. in calves is associated with airway obstruction and pulmonary inflammation. Vet. Res. 38:711-728.

Lago, A., S. M. McGuirk, T. B. Bennett, N. B. Cook, and K. V Nordlund. 2006. Calf respiratory disease and pen microenvironments in naturally ventilated calf barns in winter. J. Dairy Sci. 89:4014-4025

Lundborg, G. K., E. C. Svensson, and P. A. Oltenacu. 2005. Herd-level risk factors for infectious diseases in Swedish dairy calves aged 0-90 days. Prev. Vet. Med. 68:123-143.

McCullagh, P., and A. Nelder. 1984. Generalized linear models. Ann. Stat. 12:1589-1596.

Miller, W. M., J. W. Harkness, M. S. Richards, and D. G. Pritchard. 1980. Epidemiological studies of calf respiratory disease in a large commercial veal unit. Res. Vet. Sci. 28:267-274.
Nikunen, S., H. Härtel, T. Orro, E. Neuvonen, R. Tanskanen, S. L. Kivelä, S. Sankari, P. Aho, S. Pyorälä, H. Saloniemi, and T. Soveri 2007. Association of bovine respiratory disease with clinical status and acute phase proteins in calves. Comp. Immunol. Microbiol. Infect. Dis. 30:143-151.

Norström, M., E. Skjerve, and J. Jarp. 2000. Risk factors for epidemic respiratory disease in Norwegian cattle herds. Prev. Vet. Med. 44:87-96.

Radaelli, E., M. Luini, G. R. Loria, R. A. J. Nicholas, and E. Scanziani. 2008. Bacteriological, serological, pathological and immunohistochemical studies of Mycoplasma bovis respiratory infection in veal calves and adult cattle at slaughter. Res. Vet. Sci. 85:282-290.

Sivula, N. J., T. R. Ames, W. E. Marsh, and R. E. Werdin. 1996. Descriptive epidemiology of morbidity and mortality in Minnesota dairy heifer calves. Prev. Vet. Med. 27:155-171.

Snowder, G. D., L. D. Van Vleck, L. V. Cundiff, and G. L. Bennett. 2006. Bovine respiratory disease in feedlot cattle: Environmental, genetic, and economic factors. J. Anim. Sci. 84:1999-2008.

Somers, J. G. C. J., K. Frankena, E. N. Noordhuizen-Stassen, and J. H. M. Metz. 2005. Risk factors for digital dermatitis in dairy cows kept in cubicle houses in The Netherlands. Prev. Vet. Med. 71:11-21.

Svensson, C., and P. Liberg. 2006. The effect of group size on health and growth rate of Swedish dairy calves housed in pens with automatic milk-feeders. Prev. Vet. Med. 73:43-53.

Svensson, C., A. Linder, and S. O. Olsson. 2006. Mortality in Swedish dairy calves and replacement heifers. J. Dairy Sci. 89:4769-4777.

Svensson, C., K. Lundborg, U. Emanuelson, and S. O. Olsson. 2003. Morbidity in Swedish dairy calves from birth to 90 days of age and individual calf-level risk factors for infectious diseases. Prev. Vet. Med. 58:179-197.

Takai, T., S. Pedersen, J. O. Johnsen, V. R. Phillips, M. R. Holden, R. W. Sneath, J. L. Short, R. P. White, J. Hartung, J. Seedorf, M. Schröder, K. H. Linkert, J. H. M. Metz, P. W. G. Koerkamp, G. H. Uenk, and C. M. Wathes. 1998. Concentrations and emissions of airborne dust in livestock buildings in Northern Europe. J. Agric. Eng. Res. 70:59-77.

Thomas, A., H. Ball, I. Dizier, A. Trolin, C. Bell, J. Mainil, and A. Linden. 2002. Isolation of Mycoplasma species from the lower respiratory tract of healthy cattle and cattle with respiratory disease in Belgium. Vet. Rec. 151:472-476.

Virtala, A. M. K., Y. T. Grohn, G. D. Mechor, and H. N. Erb. 1999. The effect of maternally derived immunoglobulin $\mathrm{G}$ on the risk of respiratory disease in heifers during the first 3 months of life. Prev. Vet. Med. 39:25-37.

Virtala, A. M. K., G. D. Mechor, Y. T. Grohn, H. N. Erb, and E. J. Dubovi. 1996. Epidemiologic and pathologic characteristics of respiratory tract disease in dairy heifers during the first 3 months of life. J. Am. Vet. Med. Assoc. 208:2035-2042.

Waltner-Toews, D., S. W. Martin, and A. H. Meek. 1986. Dairy calf management, morbidity and mortality in Ontario Holstein herds. III. Association of management with morbidity. Prev. Vet. Med. $4: 137-158$. 